\title{
Politischer Führungsanspruch auf schwindender Machtbasis: Frankreichs Europapolitik unter François Hollande
}

\author{
Joachim Schild*
}

Seine Wahl habe die europäische Politik verändert und eine Neuorientierung eingeleitet, so der am 6. Mai 2012 gewählte französische Staatspräsident François Hollande. Die Zeit des einseitigen Setzens auf Haushaltskonsolidierung im Umgang mit der Eurokrise müsse ebenso ein Ende haben wie die Rolle Frankreichs als Juniorpartner Deutschlands. Seinen Wahlsieg interpretierte der Sozialist Hollande als Ausdruck eines weit über Frankreich hinaus reichenden Wunsches nach einem Politikwechsel in Richtung einer europäischen Wachstumsstrategie, die die notwendige Haushaltskonsolidierung ergänzen und erleichtern müsse. ${ }^{1}$

Wie gedenkt der neue Präsident diese hohen Ansprüche einzulösen und welche Erfolgsaussichten bestehen für eine Neuausrichtung europäischer Krisenbewältigungspolitik entlang französischer Prioritäten? Diese Fragen verweisen auf ein grundlegendes Spannungsfeld, in dem sich die französische Europapolitik immer bewegt hat, nämlich zwischen machtpolitischen Motiven und einem ausgeprägten europapolitischen Gestaltungs- und Führungswillen einerseits und begrenzten innerstaatlichen Handlungsressourcen wirtschaftlicher, finanzieller und politischer Natur andererseits. Dabei hat das Gewicht der Innenpolitik mit wachsender europäischer Integrationsdichte seit Anfang der 1990er Jahre deutlich zugenommen und europapolitische Handlungsspielräume der Exekutive tendenziell verengt, während die wirtschaftlichen Machtressourcen des Staates im letzten Jahrzehnt einen relativen Niedergang erlebt haben. ${ }^{2}$

Um die Frage nach möglichen Wegen zur Einlösung des europapolitischen Führungs- und Gestaltungsanspruchs beantworten zu können, betrachtet dieser Beitrag zunächst die politischen und wirtschaftlichen Kontextbedingungen, im Rahmen derer der neue Präsident seine Europapolitik gestaltet. Im nächsten Schritt werden die nationalen Bemühungen zur Verbesserung der wirtschaftlichen Wettbewerbsfähigkeit und zur Haushaltskonsolidierung dargestellt. Diese sind unverzichtbar zur Rekonstruktion der Machtbasis einer aktiven europapolitischen Rolle Frankreichs, aber auch für ausgewogene und konstruktive deutsch-französische Beziehungen. Daran anschließend werden die bislang erkennbaren konzeptionellen Elemente zur Bewältigung der Eurozonenkrise und die politischen Strategien zur Durchset-

* Prof. Dr. Joachim Schild, Lehrstuhl für vergleichende Regierungslehre, Universität Trier.

Die Arbeit des Autors zu Fragen der Gouvernanz der Eurozone wird von der Europäischen Kommission im Rahmen einer Jean-Monnet-Aktion aus Mitteln des Programms für Lebenslanges Lernen (LLP) unterstützt. Die Verantwortung für den Inhalt dieser Veröffentlichung trägt allein der Verfasser; die Kommission haftet nicht für die weitere Verwendung der darin enthaltenen Angaben.

1 Vgl. etwa seine Ansprache und seine Pressekonferenz am 13. November 2012 nach sechs Monaten im Amt. Présidence de la République: Ouverture de la conférence de presse, Palais de l'Elysée, 13 novembre 2012, 17 heures, abrufbar unter: http://www.ambafrance-de.org/IMG/pdf/confpresse_hollande11_12.pdf (letzter Zugriff: 23.11.2012)

2 Zum Einfluss der Innenpolitik beziehungsweise innenpolitischer Akteure auf die Gestaltung der Europapolitik vgl. Joachim Schild: Die ,Domestizierung“ französischer Europapolitik, in: Deutsch-Französisches Institut (Hrsg.): Frankreich-Jahrbuch 2008, Wiesbaden 2009, S. 29-49. Am Beispiel der Präsidentschaft Nicolas Sarkozys vgl. Joachim Schild: Sarkozys Europapolitik. Das zunehmende Gewicht der Innenpolitik, in: integration 3/2007, S. $238-250$ 
zung französischer Zielvorstellungen dargestellt. Abschließend sollen die Folgen des Machtwechsels an der Seine für die deutsch-französischen Beziehungen in der Europapolitik bewertet werden.

\section{Kontextbedingungen}

\section{Fragile politische Machtbasis}

Auf den ersten Blick könnte die innenpolitische Machtbasis für eine ambitionierte Europapolitik des neuen Präsidenten nicht besser sein. Gestützt auf ein klares Wählervotum bei den Präsidentschafts- und Parlamentswahlen stellt die politische Linke nicht nur den Präsidenten, sondern dominiert erstmals in der Geschichte der V. Republik sowohl die Nationalversammlung als auch den Senat und stellt darüber hinaus auch noch die Mehrheit in 21 von 22 Regionen Frankreichs. In der politisch entscheidenden Nationalversammlung verfügt die Fraktion der Parti socialiste (PS) gemeinsam mit den ihr angeschlossenen unabhängigen Abgeordneten sogar ohne den grünen Bündnispartner über eine absolute Mehrheit der Sitze. Gleichzeitig befindet sich der politische Rivale, die neogaullistische UMP (Union pour un Mouvement Populaire), nach der verlorenen Präsidentschaftswahl in einer tiefen Krise und durchlebte eine sehr polarisierende Auseinandersetzung um den Parteivorsitz zwischen JeanFrançois Copé und Ex-Premierminister François Fillon, die sie an den Rand einer Spaltung brachte.

Gleichwohl hat sich die innenpolitische Unterstützungsbasis für die Europapolitik des Präsidenten rasch als eher fragil herausgestellt. Die Ratifizierung des europäischen Fiskalpakts $^{3}$ ließ die Risse im innenpolitischen Fundament der Hollande'schen Europapolitik schnell zutage treten. Die Präsidentenpartei PS ist seit der traumatisierenden Erfahrung des 2005 gescheiterten französischen Referendums zum europäischen Verfassungsvertrag in europapolitischen Fragen tief gespalten. Zahlreiche sozialistische Abgeordnete und Senatoren betrachteten den Fiskalpakt und sein Kernstück, eine nationale Schuldenbremse, als Ausdruck einer von Deutschland durchgesetzten ,rechten', austeritätsfixierten, wachstumsfeindlichen und letztlich krisenverschärfenden Politik. Dies steht völlig in Übereinstimmung mit der Bewertung seitens des sozialistischen Präsidentschaftsbewerbers Hollande, der als Kandidat vehement eine Neuverhandlung des Fiskalpakts gefordert hatte. Als Präsident begnügte er sich mit dessen Ergänzung durch einen Pakt für Wachstum und Beschäftigung, der vom Europäischen Rat im Juni 2012 verabschiedet wurde. ${ }^{4}$ Es bedurfte endloser Sitzungen der sozialistischen Parlamentsfraktion und intensiver Bearbeitung vieler Abgeordneter, vor allem durch Europaminister Bernard Cazeneuve, um die Zahl der Abweichler in den eigenen Reihen in Grenzen zu halten. Gleichwohl stimmten nicht weniger als 20 Sozialisten in der Nationalversammlung und 3 sozialistische Senatoren gegen die Ratifizierung des Fiskalpakts, weitere 9 sozialistische Nationalversammlungsabgeordnete enthielten sich der Stimme. Auch die grüne Partei (Europe écologie Les Verts) zeigte sich in dieser Frage gespalten und war kein verlässlicher Verbündeter im Regierungslager. Die grüne Nationalversammlungsfraktion stimmte mit großer Mehrheit (einschließlich ihrer Fraktionsvorsitzenden) gegen den Vertrag: 3 dafür, 13 dagegen, 2 Enthaltungen. ${ }^{5}$ Somit hatte das Regierungslager in dieser wichtigen europapolitischen Frage keine eigenständige Mehrheit in Nationalver-

3 Vertrag über Stabilität, Koordinierung und Steuerung in der Wirtschafts- und Währungsunion.

4 Europäischer Rat: Tagung des Europäischen Rates 28./29. Juni 2012. Schlussfolgerungen, Euco 76/2/12, S. 7-15.

5 Vgl. Solenn de Royer: Les ministres Verts sauvent leur tête, in: lefigaro.fr, 25.9.2012. 
sammlung und Senat. Sie war zur Ratifizierung des Vertrags auf die - massiv ausgefallene - Zustimmung aus den Reihen der oppositionellen Neogaullisten und Zentristen angewiesen.

Die kontroverse Ratifizierung des Fiskalpakts hat zum wiederholten Male die gestiegene Bedeutung von Europathemen als Spaltungslinie französischer Politik verdeutlicht, eine Trennlinie, die mitten durch die aktuellen Regierungsparteien verläuft. Die Debatte um den Fiskalpakt und die darin enthaltene Verpflichtung zu einer nationalen Schuldenbegrenzungsregel hat auch erneut gezeigt, in welchem Ausmaß der aktuelle, als ,neoliberal ' und stabilitätsbesessen wahrgenommene Entwicklungspfad europäischer Integration innerhalb der französischen Linken auf grundsätzliche Bedenken stößt. Dies ist eine innenpolitische Hypothek, die die Amtszeit von Hollande dauerhaft belasten dürfte. Dieser hat sich während der gesamten Debatte um die Ratifizierung des Fiskalpakts erkennbar im Hintergrund gehalten und dem Premierminister Jean-Marc Ayrault und seiner Regierung das Feld überlassen. Diese geringe Bereitschaft des Präsidenten, politisches Kapital in die Sicherstellung einer Legitimationsbasis für seine Europapolitik zu investieren, seine Weigerung, offensiv um Zustimmung für seine Politik zu werben und eine breite und kontroverse Europadebatte zu führen, mag dem inneren Frieden seiner Partei dienen und zur Kohäsion der Präsidentenmehrheit beitragen; sie könnte sich aber angesichts anstehender schwieriger europapolitischer Grundsatzentscheidungen während seiner Amtszeit als innenpolitische Achillesferse seiner Europapolitik erweisen.

Dies gilt umso mehr, als das europapolitische Legitimations- und Akzeptanzproblem ein breites gesellschaftliches Phänomen darstellt. Eurobarometer-Umfragen vermitteln seit rund zehn Jahren das Bild eines wachsenden Euroskeptizismus und einer im Vergleich aller Mitgliedstaaten unterdurchschnittlichen Unterstützung der Europäischen Union. Eine im Wahlkampfkontext durchgeführte nationale Umfrage ließ deutliche Tendenzen des Rückzugs auf den Nationalstaat erkennen. ${ }^{6}$ Die Ursachen für die sich verschärfenden sozioökonomischen Problemlagen Frankreichs suchen viele Franzosen dabei häufig außerhalb der Grenzen des eigenen Staates - in der Globalisierung und in der europäischen Politik -, weniger dagegen in den Schwächen des eigenen Wirtschafts- und Sozialmodells. ${ }^{7}$ Ein Referendum zur innerstaatlichen Ratifizierung von möglichen Vertragsänderungen hätte derzeit in Frankreich nur sehr geringe Erfolgsaussichten.

\section{Frankreich - der , kranke Mann Europas'?}

Die wohl schwierigste Hypothek französischer Europapolitik, ja französischer Politik überhaupt, besteht in der seit Jahren zu beobachtenden rapiden Erosion französischer wirtschaftlicher Wettbewerbsfähigkeit und einer damit verbundenen Wachstumsschwäche. ${ }^{8}$

6 Befragt, welches die beste Lösung sei, um die großen Zukunftsprobleme zu bewältigen, wählten in einer IpsosUmfrage vom März 201256 Prozent der Befragten die Antwortoption ,,die Machtbefugnisse des Staates zu verstärken, selbst wenn dies diejenigen Europas begrenzt“. Die Alternative, ,die Machtbefugnisse Europas zu stärken, selbst wenn dies diejenigen des Staates beschränkt“, erschien nur 38 Prozent als attraktiv. Vgl. Le Monde: $56 \%$ des Français souhaitent renforcer les pouvoirs nationaux face à Bruxelles, 10.3.2012 (eigene Übersetzung).

7 Vgl. Philip H. Gordon/Sophie Meunier: The French Challenge: Adapting to Globalization, Washington D.C. 2001; Timothy B. Smith: France in Crisis: Welfare, Inequality, and Globalization since 1980, Cambridge 2005.

8 Einen Überblick über Wettbewerbsfähigkeitsprobleme gibt eine im Auftrag des französischen Finanzministeriums erstellte Studie des Wirtschaftsforschungsinstituts Coe-Rexecode. Vgl. Coe-Rexecode: Mettre un terme à la divergence de compétitivité entre la France et l'Allemagne. Étude réalisée pour le Ministère de l'Economie, des Finances et de l'Industrie, 14.1.2011; vgl. auch die neuere Studie Coe-Rexecode: Faiblesses et atouts de la France dans la zone euro: le défi de la reconvergence, Paris 2012. 
Diese erschwert nicht nur die angestrebte Haushaltskonsolidierung, ${ }^{9}$ sondern unterminiert zunehmend auch die wirtschaftliche Basis einer proaktiven französischen Europapolitik und damit auch den Anspruch auf eine mit Deutschland gleichberechtigte französische Führungsrolle in der Europäischen Union, insbesondere in der Bewältigung der Eurozonenkrise. Die stabilisierende Rolle des deutsch-französischen „eingebetteten Bilateralismus“10 im europäischen Integrationskontext würde längerfristig durch eine zunehmende wirtschaftliche und machtpolitische Asymmetrie zwischen den beiden Partnern deutlich erschwert, da sich Frankreich kaum mit der Rolle eines Juniorpartners anfreunden dürfte.

Zahlreiche Indikatoren belegen einen geradezu dramatisch zu nennenden Verlust an Wettbewerbsfähigkeit seit dem Start der europäischen Währungsunion 1999, insbesondere im Vergleich zum deutschen Nachbarn (siehe Tabelle 1).

Tabelle 1: Wirtschaftliche Wettbewerbsfähigkeit: Frankreich und Deutschland im Vergleich

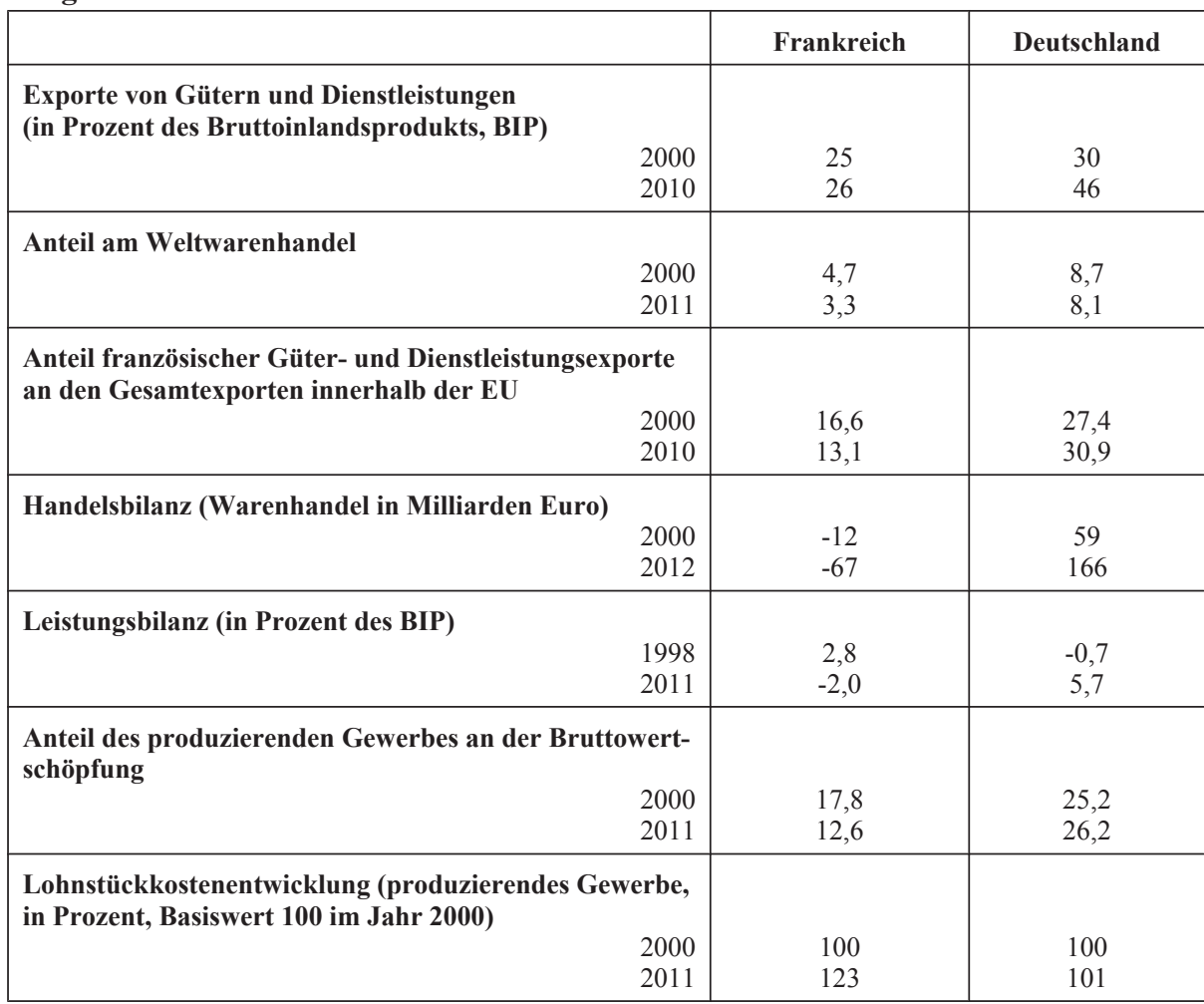

Quellen: Exporte in Prozent des BIP: OECD; Anteil am Weltwarenhandel: WTO; Handelsbilanz: Europäische Kommission: AMECO-Datenbank; Leistungsbilanz: Eurostat; Lohnstückkostenentwicklung: Coe-Rexecode: Mettre un terme à la divergence de compétitivité entre la France et l'Allemagne, 2011, S. 39 und 40; Anteil des produzierenden Gewerbes an der Bruttowertschöpfung: Destatis/Frankfurter Allgemeine Zeitung, 3.11.2012.

9 Siehe unten.

10 Vgl. hierzu: Ulrich Krotz/Joachim Schild: Shaping Europe. France, Germany, and Embedded Bilateralism from the Elysée Treaty to Twenty-First Century Politics, Oxford 2013. 
Ein stark sinkender Anteil französischer Exporte am Welthandel und wachsende Handelsund Leistungsbilanzdefizite bestimmen das Bild. Im Jahr 2010 exportierten die Niederlande mit ihren knapp 17 Millionen Einwohnern mehr Waren als Frankreich mit seinen rund 63 Millionen Einwohnern (Niederlande: 474 Milliarden Euro; Frankreich 429 Milliarden Euro; zum Vergleich: Deutschland: 1.058 Milliarden Euro). ${ }^{11}$

Besonders beunruhigend ist der rapide Verlust an industrieller Substanz. ${ }^{12}$ Im letzten Jahrzehnt gingen rund 750.000 Industriearbeitsplätze verloren. Der Anteil des produzierenden Gewerbes an der Gesamtwertschöpfung sank rapide von 17,8 im Jahr 2000 auf nur noch 12,6 Prozent im Jahr 2011, während er in Deutschland im gleichen Zeitraum von 25,2 auf 26,2 Prozent leicht anstieg (EU-Durchschnitt: 19,5 Prozent). ${ }^{13}$ Zudem leidet die französische Wirtschaft derzeit unter einem ,historisch niedrigen Niveau von Unternehmensprofitabilität“, ${ }^{14}$ das die Investitions- und Innovationskraft der Unternehmen unterminiert. ${ }^{15}$ „Ohne entschiedenes Handeln auf der Angebotsseite wird Frankreich, eingeklemmt zwischen einem leistungsfähigen Nordeuropa und einem sich reformierenden Südeuropa, schnell die schon gut gefüllten Ränge der Kranken Europas verstärken“, warnt der französische Ökonom und Leiter des Brüsseler Think-Tanks Bruegel, Jean Pisani-Ferry. ${ }^{16}$

Nach einem verlorenen Jahrzehnt mit nur halbherzigen Reformen während der Präsidentschaften der Neogaullisten Jacques Chirac und Nicolas Sarkozy tritt der amtierende Präsident Hollande ein besonders schweres Erbe an. ${ }^{17}$ Einer entschlossenen Reformpolitik zur Senkung der Lohn(-neben-)kosten, zur Flexibilisierung von Arbeitsmärkten und zugunsten einer strukturellen Verbesserung der Angebotsbedingungen für die französischen Unternehmen stehen dabei Dogmen und mentale Blockaden auf der politischen Linken entgegen, etwa die 35-Stunden-Woche, der hohe Mindestlohn (SMIC) und das rigide Arbeits- und Kündigungsschutzrecht.

Nach massiven Steuererhöhungen für Unternehmen und Bezieher hoher Einkommen im Haushaltsgesetz für 2013 hat die Regierung im November 2012 einen ,nationalen Pakt für Wachstum, Wettbewerbsfähigkeit und Beschäftigung“ auf den Weg gebracht. Er sieht deutliche Steuerentlastungen für Unternehmen vor, beginnend im Jahr 2013. Damit befolgt die Regierung zumindest in der Tendenz die Ratschläge einer von Hollande eingesetzten Expertenkommission unter Leitung des früheren SNCF- ${ }^{18}$ und EADS-Managers ${ }^{19}$ Louis Gallois. ${ }^{20}$

Die umfangreichen Steuerentlastungen für Unternehmen in Höhe von jährlich 20 Milliarden Euro müssen vor dem Hintergrund der im Haushaltsgesetz für 2013 beschlossenen

11 Vgl. Eurostat: Handelsbilanzdefizit des Euroraums bei 7,6 Mrd. Euro Defizit von 23,8 Mrd. Euro für die EU27, Pressemitteilung, 16.3.2012, STAT/12/44.

12 Vgl. hierzu Patrick Artus/Marie-Paule Virard: La France sans ses usines, Paris 2011.

13 Selbst südeuropäische Staaten wie Spanien (16,9 Prozent) und sogar Griechenland (13,3 Prozent) weisen höhere Anteile der Industrie an der Bruttowertschöpfung aus als Frankreich. Vgl. Frankfurter Allgemeine Zeitung: Industrie bringt 26 Prozent der Wirtschaftsleistung, 3.11.2012.

14 So die Europäische Kommission: European Economic Forecast. Autumn 2012, European Economy 7/2012.

15 Die Gewinnmargen französischer Unternehmen außerhalb des Finanzsektors liegen mit 28 Prozent auf ihrem niedrigsten Niveau seit Mitte der 1980er Jahre und bilden ,die Achillesferse der französischen Wirtschaft“, so die Großbank BNP Paribas in einer Studie. Vgl. BNP Paribas France. Growth at standstill, Paris, SeptemberOktober 2012, S. 15.

16 Vgl. Jean Pisani-Ferry: Compétitivité des entreprises: les trois débats, in: Le Monde, 6.11.2012.

17 Einen guten Überblick bietet Henrik Uterwedde: Zeit für Reformen. Frankreichs Wirtschaft im Wahljahr, Deutsche Gesellschaft für Auswärtige Politik: DGAP-Analyse Nr. 5, April 2012.

18 Société nationale des chemins de fer français.

19 European Aeronautic Defence and Space Company.

20 Diese hatte massive Entlastungen der Unternehmen von Lohnnebenkosten gefordert. Vgl. Louis Gallois: Pacte pour la compétitivité de l'industrie française, Rapport au Premier ministre, Paris, 5.11.2012. 
erheblichen staatlichen Einnahmeerhöhungen von 20 Milliarden Euro gesehen werden rund 10 Milliarden davon lasten auf Unternehmen. Diese schaffen ein ,investitionsfeindliches Umfeld“", wie der deutsche Sachverständigenrat in besorgter Diktion warnt. ${ }^{21}$

In einer optimistischen Interpretation kann der von der Regierung Ayrault beschlossene Maßnahmenkatalog zur Wiedergewinnung französischer Wettbewerbsfähigkeit ${ }^{22}$ als Schwenk hin zu einer angebotsorientierten Wirtschaftspolitik gewertet werden. Finanzminister Pierre Moscovici qualifizierte den französischen Wettbewerbspakt gar als „kopernikanische Revolution“. ${ }^{23}$ Eine deutliche Neuorientierung ist gewiss im klaren Eingeständnis von Kostenproblemen der Unternehmen zu erkennen, kaum jedoch in Umfang und Reichweite der angekündigten Entlastungsmaßnahmen.

Präsident Hollande hat seine gesamte Amtszeit unter das Motto der Wiedereroberung („reconquête“) von Wettbewerbsfähigkeit und Beschäftigung gestellt. ${ }^{24}$ Es bleibt abzuwarten, ob damit ein echter Paradigmenwechsel der französischen Wirtschaftspolitik eingeleitet wird, wie er unter François Mitterrand 1983 vollzogen wurde. Dieser bildete damals die entscheidende Voraussetzung für eine aktive französische Europapolitik und eine fruchtbare Phase des deutsch-französischen Bilateralismus in der Europäischen Union in den Folgejahren.

Ohne entschlossene Reformen droht Frankreich mittelfristig zum Problemfall der Eurozone zu werden. ${ }^{25}$ Noch unter Präsident Sarkozy stufte die Ratingagentur Standard \& Poor's im Januar 2012 das Kreditrating für französische Staatsschuldtitel von AAA auf AA+ herab. Die Agentur Moody's zog am 19. November 2012 nach und setzte Frankreichs Bonität ebenfalls auf die zweitbeste Bewertung (von 20) herab - mit negativem Ausblick. Kurze Zeit später hat Moody's mit Verweis auf Frankreich am 30. November 2012 auch das Kreditrating der Europäischen Finanzstabilisierungsfazilität (EFSF) und des Europäischen Stabilitätsmechanismus (ESM) herabgestuft. Bisher genießt Frankreich noch historisch günstige Zinsbedingungen dank minimaler Risikoaufschläge für seinen Staatsschuldenberg. Dies könnte sich aber ändern. Kein europäischer Rettungsfonds könnte einer Spekulationswelle gegen Frankreich Einhalt gebieten.

\section{Haushaltskonsolidierung als Priorität}

Präsident Hollande und die Regierung Ayrault sind sich im Klaren darüber, dass es Zeit braucht, um das Vertrauen der Märkte in die Reformfähigkeit des Staates zurückzugewinnen. Umso dringlicher sind energische Schritte zur Haushaltskonsolidierung. Schon als Präsidentschaftsbewerber ließ Hollande keinen Zweifel daran aufkommen, dass die Haushaltskonsolidierung eine zentrale Priorität seiner Finanzpolitik darstellt. Der Haushalt 2013 sowie die Haushaltsplanung bis 2017 lassen erkennen, dass es der neuen Exekutive mit der Verwirklichung dieses Ziels ernst ist. Dies unterscheidet ihn von seinem Vorgänger Sarkozy, der sich unmittelbar nach Amtsantritt über die von Frankreich auf europäischer Ebene eingegangenen Verpflichtungen zur Haushaltskonsolidierung hinwegsetzte.

21 Vgl. Sachverständigenrat zur Begutachtung der gesamtwirtschaftlichen Entwicklung: Stabile Architektur für Europa - Handlungsbedarf im Inland. Jahresgutachten 2012/13, Wiesbaden, November 2012.

22 Vgl. Premier ministre: Pacte national pour la croissance, la compétitivité et l'emploi, 6.11.2012, Paris.

23 Vgl. Marie Visot: Moscovici: Le pacte de compétitivité est une révolution copernicienne pour la gauche, Interview mit Pierre Moscovici, in: Le Figaro, 7.11.2012.

24 Vgl. Présidence de la République: Ouverture de la conférence de presse, 2012.

25 Vgl. hierzu den Frankreich-Länderreport des Economist unter einem provozierenden Titel. The Economist: The time-bomb at the heart of Europe, 17.-23.11.2012. 
Die neue Regierung will bis 2017 ein ausgeglichenes Budget erreichen - mit den beiden Zwischenzielen eines Defizits von 4,5 Prozent in 2012 und 3 Prozent in 2013. Diese Planung beruht allerdings auf unrealistischen Wachstumserwartungen. Zudem beschritt die Regierung im Haushalt 2013 den Weg, die Konsolidierung ganz überwiegend über die Einnahmenseite mittels massiver Steuererhöhungen zu erreichen (im Umfang von circa 20 Milliarden Euro), während die Ausgabenseite einen ungleich geringeren Beitrag liefern soll (circa 10 Milliarden Euro). Die Erhöhung der Steuerbelastung in einem Staat, dessen Staatsquote knapp 57 Prozent beträgt, dürfte wachstumshemmend wirken. Damit verschärft sich das Dilemma für Präsident Hollande. Um einen Vertrauensverlust auf internationalen Finanzmärkten zu vermeiden und keine deutliche Verschlechterung der Refinanzierungskonditionen für die französische Staatsschuld sowie neuerliche Turbulenzen in der Eurozone zu riskieren, muss er an der Politik der Haushaltskonsolidierung festhalten und die Sparpolitik in den nächsten Jahren eventuell verschärfen - und damit potenziell rezessive Tendenzen verstärken. Für die kommenden Jahre hat Präsident Hollande angekündigt, dass Schritte der Haushaltssanierung nicht mehr durch Steuererhöhungen, sondern im Wesentlichen über Ausgabenreduzierung erfolgen sollen, die weniger wachstumshemmend sein dürften.

Von entscheidender Bedeutung wird sein, ob es Frankreich gelingt, die rasante Erhöhung seiner Schuldenquote (Gesamtverschuldung im Verhältnis zum Bruttoinlandsprodukt) zu stoppen (siehe Tabelle 2). Ein über Jahre zu verfolgender konsequenter Konsolidierungskurs dürfte die Solidität der präsidentiellen Mehrheit und die Legitimationsbasis seiner Europapolitik einer schweren Belastungsprobe aussetzen.

Tabelle 2: Entwicklung von öffentlichem Defizit und Gesamtverschuldung 2009-2014

\begin{tabular}{|l|c|c|c|c|c|c|}
\hline & $\mathbf{2 0 0 9}$ & $\mathbf{2 0 1 0}$ & $\mathbf{2 0 1 1}$ & $\mathbf{2 0 1 2}$ & $\mathbf{2 0 1 3}$ & $\mathbf{2 0 1 4}$ \\
\hline $\begin{array}{l}\text { Schuldenquote } \\
\text { (in Prozent des } \\
\text { BIP) }\end{array}$ & 79,2 & 82,3 & 86,0 & 90,0 & 92,7 & 93,8 \\
\hline $\begin{array}{l}\text { Defizitquote } \\
\text { (in Prozent des } \\
\text { BIP) }\end{array}$ & $-7,5$ & $-7,1$ & $-5,2$ & $-4,5$ & $-3,5$ & $-3,5$ \\
\hline
\end{tabular}

Quelle: Europäische Kommission: European Economic Forecast. Autumn 2012, European Economy 7/2012. (Zahlen ab 2012 beruhen auf vorläufigen Schätzungen und Prognosen der Kommission).

\section{Umgang mit der Eurokrise}

Der Umgang mit der Staatsschulden- und Bankenkrise der Eurozone wird den Schwerpunkt der französischen Europapolitik der kommenden Jahre bilden. Auf diesem Feld lassen sich deutliche Kontinuitätslinien zur Politik des Vorgängers Sarkozy erkennen, aber auch eine Reihe neuer Elemente. Kontinuität ist im Hinblick auf die gewünschte Stärkung der europäischen Interventionsmechanismen zur Unterstützung einzelner Eurozonenmitglieder erkennbar. Hollande dringt genau wie sein Vorgänger auf eine entschlossene Bekämpfung von spekulativen Dynamiken mittels der Bereitstellung umfangreicher Liquidität für Krisenstaaten. Dieses Ziel könnte entweder über die EFSF beziehungsweise den ESM oder über entschlossene Interventionen der Europäischen Zentralbank (EZB) erreicht werden. Seine Wahlkampfforderung nach Änderung der EZB-Satzung, um ihr eine Rolle als „Gläubiger 
der letzten Instanz“ zuschreiben zu können, ${ }^{26}$ hat er nach der Wahl nicht aktiv eingebracht. Dies hätte die deutsch-französischen Beziehungen gewiss belastet. Die Idee, dem ESM eine Banklizenz zu verleihen, um ihm einen Rückgriff auf EZB-Liquidität zu ermöglichen, unterstützte er jedoch auch nach seiner Wahl. ${ }^{27}$

Kontinuität ist auch im Hinblick auf das Ziel zu erkennen, Griechenlands Verbleib in der Eurozone zu sichern, selbst wenn diese mit erheblichen Mehrkosten verbunden sein sollte. Auch das entschlossene Eintreten von Präsident und Regierung für Schritte in Richtung einer Bankenunion steht durchaus in der Logik der Politik ihrer Vorgänger. So machte sich Hollande mit Erfolg für die Möglichkeit einer direkten Bankenrekapitalisierung durch den ESM stark - ohne den Umweg über nationale Haushalte -, um die wechselseitigen Verstärkungseffekte zwischen Banken- und Staatsschuldenkrisen zu verringern. In einer umfassenden Bankenunion mit europäischen Aufsichtsstrukturen, der Möglichkeit, krisengeschüttelte Banken zu rekapitalisieren oder abzuwickeln sowie mit einem europäischen Bankeinlagensicherungssystem sieht Frankreich den zentralen nächsten Schritt im Rahmen der Bewältigung der Eurozonenkrise. ${ }^{28}$ Entsprechend hat sich Hollande auf dem Europäischen Rat vom 18./19. Oktober 2012 mit großem Nachdruck für einen engen Zeitplan zur Verwirklichung einer europäischen Bankenaufsicht eingesetzt, die von der Bundesregierung zur Voraussetzung für die Nutzung von ESM-Mitteln zur direkten Bankenrekapitalisierung gemacht wurde. Allerdings besitzen derzeit weder die Einrichtung eines Fonds zur Bankenabwicklung noch ein europäisches Einlagensicherungssystem große Realisierungschancen.

In zwei wichtigen Punkten - Eurobonds und Fiskalpakt - unterscheidet sich der neue Präsident jedoch von seinem Vorgänger - und von der bundesdeutschen Position. Hollande verfolgt die Idee von Eurobonds, einer gemeinsamen Ausgabe von Staatsschuldtiteln in der Eurozone und gemeinsamer Haftung für Ausfallrisiken, die Sarkozy nicht aktiv propagierte. „Die deutsche Haltung ist es, optimistisch betrachtet, Eurobonds als Zielperspektive zu sehen, während sie für uns ein Ausgangspunkt sind“", so der neugewählte Staatspräsident. ${ }^{29} \mathrm{Er}$ brachte sogar eine Vergemeinschaftung von Altschulden ins Spiel, also nicht nur die Ausgabe neuer Schuldtitel in Form von Eurobonds. ${ }^{30}$ Der französische Finanzminister Moscovici äußerte jedoch Verständnis dafür, dass die Bundesregierung in dieser Frage eine rote Linie ziehe. $^{31}$

Der Umgang mit dem Fiskalpakt bildet den zweiten wichtigen Punkt, in dem sich Hollande von seinem Vorgänger unterscheidet. Im Wahlkampf legte er sich darauf fest, den Fiskalpakt

26 Vgl. Frankfurter Allgemeine Zeitung: Hollande kritisiert Fiskalpakt, 19.3.2012.

27 Vgl. seine gemeinsame Pressekonferenz mit dem spanischen Ministerpräsidenten Rajoy vom 23. Mai 2012 in Paris. François Hollande/Mariano Rajoy: Conférence de presse conjointe du président de la république, M. François Hollande et du président du gouvernement du royaume d'Espagne, M. Mariano Rajoy - Propos de M. Hollande, abrufbar unter: http://basedoc.diplomatie.gouv.fr/exl-doc/EPI00054597.pdf (letzter Zugriff: 21.11.2012).

28 Vgl. die Rede von Finanzminister Pierre Moscovici vor dem Brüsseler Think-Tank Bruegel am 7. September 2012. Bruegel: Pierre Moscovici's speech at Bruegel Annual Meeting, 7.9.2012, abrufbar unter: www.bruegel.org/nc/blog/detail/article/883-pierre-moscovicis-speech-at-bruegel-annual-meeting (letzter Zugriff: 12.11.2012).

29 Zitiert nach Le Monde: Autour de la table du Conseil européen, les camps mettent à plat leurs arguments, 25.5.2012.

30 Vgl. Discours de M. le Président de la République $-X^{\text {ème }}$ Conférence des Ambassadeurs, Paris, 27. August 2012.

31 Vgl. Handelsblatt.com: Berlin und Paris kündigen „komplette Lösung“ an, 30.10.2012. 
neu zu verhandeln (,renégocier"). ${ }^{32}$ Dies war in Berlin auf entschiedenen Widerstand gestoßen, wo man auf das Prinzip des Pacta sunt servanda europäischer Politik verwies. ${ }^{33}$

Hollande gelang es auf seinem ersten Europäischen Rat am 28./29. Juni 2012, die Ergänzung des Fiskalpakts durch einen europäischen „Pakt für Wachstum und Beschäftigung“ durchzusetzen, ohne jedoch den Vertrag über ersteren aufzuschnüren. Die im Wachstumspakt gebündelten Maßnahmen in einem Gesamtumfang von 120 Milliarden Euro wie etwa eine Pilotphase für europäische Projektbonds, die Nutzung nicht ausgeschöpfter Strukturfondsgelder und eine Kapitalerhöhung der Europäischen Investitionsbank dürften kaum mehr als recht überschaubare Nachfrage- und Wachstumsimpulse zur Folge haben, zumal wenig neue Gelder hierfür vorgesehen sind. Ein Paradigmenwechsel im Management der Eurozonenkrise, wie ihn der französische Präsident dem heimischen Publikum glaubhaft zu machen versuchte, ${ }^{34}$ ist damit keineswegs verbunden.

\section{„Solidarische Integration “}

Präsident Hollande fasst die von ihm massiv befürwortete Stärkung von Elementen der Risikoteilung und wechselseitigen Haftung zwischen Mitgliedstaaten in Form von Rettungsfonds, Eurobonds, einer europäischen Bankeneinlagensicherung sowie einer aktiven Kriseninterventionspolitik der EZB unter dem Begriff der „,solidarischen Integration“ zusammen. Diese hat er zum Leitmotiv seiner Europapolitik gemacht. Den schon bekannten Forderungen nach mehr „Feuerkraft“ zum Löschen von akuten Brandherden fügte die französische Exekutive eine neue Idee hinzu. In einer Rede vor der Brüsseler wirtschaftswissenschaftlichen Denkfabrik Bruegel deutete Finanzminister Moscovici Konturen eines fiskalischen Föderalismus als längerfristige Perspektive für die Europäische Union an. ${ }^{35}$ Ein eigenständiges Budget der Eurozone, gespeist aus eigenständigen Steuerquellen sowie durch Eurobonds, könnte europäische politische Prioritäten finanzieren. Als Beispiel für politische Schwerpunktsetzungen nannte er europäische Unterstützungsleistungen für Arbeitslose. Damit gab er zum einen der Diskussion um Eurobonds eine andere Stoßrichtung, sieht sein Vorschlag diese doch zur Finanzierung europäischer politischer Prioritäten und eines Eurozonenbudgets vor, nicht zum Stopfen nationaler Haushaltslöcher. Das Beispiel der Finanzierung von Leistungen für Arbeitslose verweist zudem auf eine makroökonomische Stabilisierungsfunktion eines Eurozonenbudgets dank der Wirkung als automatischer Nachfragestabilisator in Krisenzeiten. Das Plädoyer des Präsidenten zugunsten einer Vergemeinschaftung von Altschulden unterstreicht jedoch, dass Frankreich Eurobonds weiterhin auch im Sinne einer gemeinsamen Haftung für nationale Schulden und somit als Instrument der Krisenbekämpfung und Beruhigung der Finanzmärkte betrachtet.

Die gesamte Stoßrichtung der französischen Vorschläge in Richtung einer solidarischen Integration wirft zwei grundsätzliche Fragen auf: Werden die innereuropäische Solidaritätsbereitschaft und die Legitimationsressourcen für die vorgeschlagenen Vertiefungsschritte von der französischen Führung nicht dramatisch überschätzt? Und wie ist das Verhältnis zwischen wechselseitiger Solidarität und wechselseitiger Kontrolle gedacht?

Die von Frankreich eingeforderte verstärkte „solidarische Integration“ wird nicht ohne eine Stärkung von Kontrollmechanismen und europäischer Durchgriffsrechte auf nationale

32 So Hollande in einem Namensartikel in Le Monde: Une autre voie pour l'Europe, 17.12.2011.

33 Vgl. FAZ.net: Merkel: Fiskalpakt wird nicht neu verhandelt, 7.5.2012.

34 Vgl. seine Ansprache an die Franzosen vom 13. November 2012. Présidence de la République: Ouverture de la conférence de presse, 2012.

35 Ich danke Jean-François Jamet, der mich auf diesen Punkt hingewiesen hat. 
Wirtschafts- und Haushaltspolitik zu haben sein. Hollande drehte den Spieß gleichsam um: „Ohne Solidarität kann es keine Integration, das heißt Souveränitätstransfer“ geben. ${ }^{36}$ In der aktuellen Debatte über eine politische Union als (föderaler) Rahmen der Wirtschafts- und Währungsunion hält sich der französische Präsident eher bedeckt. Im Wahlkampf hatte er föderalistischen institutionellen Blaupausen einen „Föderalismus der Projekte“ entgegengesetzt. ${ }^{37}$ Nach seiner Wahl wartet er offenbar zunächst ab, wie das Schlagwort von der ,politischen Union“ von deutscher Seite mit konkreten Inhalten gefüllt wird.

Tiefe und direkte Eingriffsrechte europäischer Organe in die nationale Haushaltspolitik scheinen für Paris zumindest derzeit nicht zustimmungsfähig zu sein. Genau wie Sarkozy verteidigt Hollande ganz in der republikanischen Tradition Frankreichs sowohl den Primat der Politik - gewährleistet über Entscheidungen im Rat - als auch nationale Souveränitätsvorbehalte. „Niemals“, so der Wahlkämpfer Hollande, ,,werde ich zulassen, dass im Namen der haushaltspolitischen Koordinierung der Europäische Gerichtshof die Ausgaben und Einnahmen eines souveränen Staates beurteilen darf.“38 Auch der von Bundesfinanzminister Wolfgang Schäuble ins Spiel gebrachte und von Angela Merkel unterstützte Vorschlag, den europäischen Währungskommissar mit haushaltspolitischen Durchgriffsrechten auszustatten, sodass ,ein Haushalt für ungültig erklärt werden könnte und dies auch vom Europäischen Gerichtshof überprüft werden könnte" ${ }^{39}$ fand keine Unterstützung seitens der französischen Exekutive. Der entscheidende Punkt aus Sicht der Bundesregierung dürfte eine wirksame ex-ante-Kontrolle nationaler Haushaltspolitik sein, da sich eine ex-post-Sanktionierung auf der Basis des Regelwerks des Stabilitäts- und Wachstumspakts in der Vergangenheit als politisch nicht durchsetzbar erwiesen hat. Zwischen der französischen Forderung nach Eurobonds und der von Deutschland geforderten Akzeptanz von ex-ante-Haushaltskontrolle durch die europäischen Organe besteht ein unauflöslicher politischer Zusammenhang. ${ }^{40}$

Hollande möchte jedoch der nationalen Haushaltspolitik keine zu engen rechtlichen Bindungen auferlegen. Dies kommt darin zum Ausdruck, dass er - im Gegensatz zu seinem Vorgänger Sarkozy - der im Fiskalpakt festgeschriebenen Verpflichtung zum Haushaltsausgleich keinen Verfassungsrang verleihen wollte. Erleichtert wurde dies durch ein Urteil des von ihm angerufenen Verfassungsrates (Conseil constitutionnel). Dieser konnte in seiner Entscheidung zur innerstaatlichen Ratifizierung des Fiskalpakts keinen zwingenden Verfassungsänderungsbedarf erkennen. ${ }^{41}$ In seinem Interview zum Nationalfeiertag am 14. Juli 2012 betonte der Präsident, dass er keine Notwendigkeit sehe, ein Engagement - die Schuldenregel - in Marmor zu meißeln, das „nur für ein paar Jahre“ gebraucht werde. ${ }^{42}$

Statt mittels eines verfassungsändernden Gesetzes wurde diese völkerrechtliche Verpflichtung mittels eines Organgesetzes im nationalen Recht verankert. Dieses ist in der Normenhierarchie zwischen der Verfassung und einfachen Gesetzen angesiedelt. Es besitzt Bin-

36 Vgl. seine beiden Pressekonferenzen im Rahmen des Europäischen Rates vom 28. und 29.6.2012, abrufbar unter: www.elysee.fr. (letzter Zugriff: 30.6.2012; eigene Übersetzung).

37 Vgl. Handelsblatt.com: Berlin und Paris kündigen „komplette Lösung“ an, 2012.

38 Frankfurter Allgemeine Zeitung: Hollande: EU darf Frankreichs Haushaltspolitik nicht beurteilen, 2.12.2011.

39 Vgl. Die Regierungserklärung von Bundeskanzlerin Merkel zum Europäischen Rat am 18. und 19. Oktober in Brüssel vor dem Deutschen Bundestag am 18. Oktober 2012, in: Deutscher Bundestag: Stenografischer Bericht. 198. Sitzung. Berlin, Donnerstag, den 18. Oktober 2012, Plenarprotokoll 17/198, S. 23810 D-23817 D.

40 Vgl. hierzu Jean Pisani-Ferry: Le réveil des démons. La crise de l'euro et comment nous en sortir, Paris 2011, S. $179-186$

41 Vgl. Conseil Constitutionnel, Décision n² 2012-653 DC du 09 août 2012.

42 Interview François Hollandes zum Nationalfeiertag, 14.7.2012, abrufbar unter: www.elysee.fr/president/root/ bank/pdf/president-13612.pdf (letzter Zugriff: 14.11.2012). 
dewirkung im Verhältnis zu den jährlichen Haushaltsgesetzen, kann aber mit der Mehrheit der Nationalversammlungsmitglieder geändert oder aufgehoben werden.

Der französische Präsident hat eine klare zeitliche Abfolge für die nächsten Etappen zur Bewältigung der Eurozonenkrise definiert. Bis Ende 2012 sollte die Europäische Union zentrale Weichenstellungen vornehmen, um den Verbleib Griechenlands in der Eurozone sowie ein niedriges Zinsniveau für Programmstaaten sicherzustellen, und die legislativen Grundlagen für eine europäische Bankenaufsicht schaffen. Ab 2013 könne dann die Debatte über eine Vertiefung der Union und eine Änderung ihrer Entscheidungsverfahren geführt werden. „Die politische Union wird nach der Haushalts-, Banken- und Sozialunion kommen. Sie wird der solidarischen Integration einen demokratischen Rahmen geben. [...] Nach den Europawahlen 2014. “43 Solidarität sofort, politische Integration (eventuell) später, so lässt sich diese Strategie zusammenfassen.

Einige Konturen der französischen Vorstellungen zur zukünftigen Union sind inzwischen durchaus erkennbar geworden. Dies gilt insbesondere im Hinblick auf die institutionelle Vertiefung der Eurozone. Paris plädiert für die Stärkung des Präsidenten der Eurogruppe. Dieser sollte ein klares Mandat und eine längere Amtszeit erhalten. Zudem könnte die Governance der Eurozone durch monatliche Treffen der Eurozonenmitglieder aufChefebene (Eurogipfel) gestärkt werden. Die institutionelle Eigenständigkeit der Eurozone ist natürlich auch in der oben erwähnten Forderung nach einem eigenständigen Budget für die Eurozonenmitglieder angelegt. Dieses könne, so Hollande, über den Weg einer verstärkten Zusammenarbeit erreicht und durch die Einnahmen der 2013 einzuführenden Finanztransaktionssteuer gespeist werden. ${ }^{44}$

Diese Vorschläge lassen die französische Präferenz zugunsten von Formen variabler Geometrie europäischer Integration in Form institutionell ausdifferenzierter Gruppenbildungsprozesse erkennbar werden. Die Eurozone bildet das primäre und prioritäre Anwendungsfeld dieser Integrationsmethode. Schritte zur Integrationsvertiefung in der Eurozone durch Teilgruppenbildung unter Nutzung des sekundärrechtlichen Instruments der verstärkten Zusammenarbeit (beispielsweise die Finanzmarkttransaktionssteuer) oder außerhalb des Unionsrechts (Fiskalpakt) haben den Vorteil, dass Frankreich langwierige europäische Vertragsreformen vermeiden könnte. Größere Reformen der Unionsverträge werfen unweigerlich die Frage nach einer Ratifikation durch das Volk in Form eines Referendums auf. Nach Lage der Dinge ist ein positiver Ausgang eines Europareferendums in Frankreich eher unwahrscheinlich. Hierin liegt eine zentrale Hürde für jede europäische Vertragsreformpolitik, die über kleinere, in einem vereinfachten Vertragsrevisionsverfahren zu verabschiedende Änderungen hinausginge.

\section{Folgen für die deutsch-französischen Beziehungen}

In Bezug auf die europapolitische Kernfrage der Amtszeit Hollandes, die Bewältigung der Staatsschulden- und Bankenkrise, bestehen erhebliche konzeptionelle Unterschiede zwischen Frankreich und Deutschland fort. Diese sind in manchen Punkten (Eurobonds) sogar noch gewachsen. Die Vorstellungen einer ,solidarischen Integration“ und einer europäischen Wirtschaftsregierung à la Hollande liegen weit entfernt von bundesdeutschen Präferenzen

43 François Hollande: Comment guérir l'Europe, in: Le Monde, 18.10.2012. Die deutsche Fassung des Interviews wurde veröffentlicht in Süddeutsche Zeitung: Geliebte Baustelle, 18.10.2012.

44 Vgl. Conseil Constitutionnel, Décision n²012-653 DC du 09 août 2012. 
und konkreten Positionen zur Entwicklung einer regelbasierten und souveränitätsbeschränkenden Stabilitätsunion.

Diese deutsch-französischen Differenzen betreffen grundsätzliche Fragen der zukünftigen Architektur der Wirtschafts- und Währungsunion.

1. Im Hinblick auf die gemeinschaftliche Haftung für nationale Schulden ist eine deutschfranzösische Annäherung allenfalls mittelfristig denkbar. Die zentrale Bedingung hierfür wäre, dass Frankreich glaubwürdige und souveränitätsbeschränkende ex ante-Kontrollen und Durchgriffsrechte der europäischen Ebene auf nationale Haushaltspolitik akzeptiert. Die Äußerungen von Präsident und Regierungsmitgliedern lassen diese Bereitschaft bislang nicht erkennen.

2. Die Bedeutung, die ,moral hazard'-Problemen und - damit auf das Engste verbunden Marktkräften für die Korrektur einer nicht nachhaltigen Fiskalpolitik beigemessen wird, unterscheidet sich erheblich. Während Paris eine differenzierte Bewertung von Bonitätsrisiken durch die Märkte als krisenverschärfend wahrnimmt und Maßnahmen zur Annäherung der Risikoprämien auf Staatsanleihen begrü $3 \mathrm{t},{ }^{45}$ betont die Bundesregierung sowohl die Bedeutung von ,moral hazard'-Problemen als auch die Rolle des Marktes im Prozess der Korrektur verfehlter nationaler Haushaltspolitik ungleich stärker.

3. Auch unterscheiden sich die Konzeptionen im Hinblick auf das notwendige Ausmaß europäischer Konvergenz und Harmonisierung im Rahmen einer Währungsunion erheblich. Die Merkel-Regierung dringt auf Strukturreformen in nationaler Verantwortung, möchte eine wirtschaftspolitische und wirtschaftliche Konvergenz in Richtung der erfolgreichsten Ökonomien im Euroraum befördern und die Eurozone damit ,deutscher' machen. Die französische Exekutive betont hingegen die Notwendigkeit partieller Harmonisierungsschritte beziehungsweise von Mindeststandards auf europäischer Ebene, etwa in der Steuer-, Sozial- und Umweltpolitik, nicht zuletzt um den Anpassungs- und Reformdruck auf das eigene Wirtschafts- und Sozialsystem zu vermindern. ${ }^{46}$ Politökonomen fassen dies unter dem Begriff einer, raising rival's costs'-Strategie.

Diese grundlegenden konzeptionellen Unterschiede zwischen beiden Staaten sind auch in der Diskussion der möglichen Funktion und Ausgestaltung eines eigenständigen Eurozonenbudgets offen zu Tage getreten. Die Bundesregierung möchte dieses Instrument nutzen, um finanzielle Anreize für nationale Strukturreformen anbieten zu können, zu denen sich die Krisenstaaten in verbindlicher Form gegenüber der Unionsebene verpflichten müssten. Paris hingegen sieht darin eher ein Instrument zur Finanzierung einer europäischen Wachstumspolitik oder zur Konjunkturstabilisierung.

Diese - keineswegs neuen - konzeptionellen Unterschiede zwischen Frankreich und Deutschland dürften im Rahmen der Weiterentwicklung der Wirtschafts- und Währungsunion immer wieder zu Tage treten. Kompromisse zwischen unterschiedlichen Ausgangspositionen, für die Frankreich und Deutschland stellvertretend stehen, sind auch in den kommenden Jahren unverzichtbar. Auch in Zukunft wird Deutschland und Frankreich aufgrund ihres ökonomischen Gewichts in der Eurozone eine Schlüsselrolle in europäischen Ent-

45 Vgl. die Pressekonferenz von François Hollande während des Europäischen Rates vom 18.-19.10.2012, abrufbar unter: www.elysee.fr. (letzter Zugriff: 21.10.2012).

46 Ein vom Europaausschuss der Nationalversammlung verabschiedeter Entschließungsantrag plädiert für eine ,ambitionierte Wachstums-, Beschäftigungs- und Investitionspolitik, gestützt auf eine weitreichende (,profon-

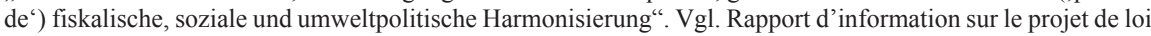
de ratification du Traité sur la stabilité, la coordination et la gouvernance au sein de l'Union économique et monétaire, présenté par Christophe Caresche, Paris, Assemblée nationale, 25. September 2012, S. 76 (eigene Übersetzung). 
scheidungsprozessen zufallen. Für die Qualität des deutsch-französischen Verhältnisses ist dabei die Frage entscheidend, wie Präsident Hollande und seine Regierung (und natürlich auch die Merkel-Regierung) mit den divergierenden Präferenzen umgehen.

Bislang ist Staatspräsident Hollande sichtbar um enge Abstimmungsprozesse jenseits des deutsch-französischen Bilateralismus bemüht. Seine zahlreichen Reisen in europäische Hauptstädte, insbesondere die engen Kontakte nach Italien und Spanien, verfolgen das Ziel, Frankreich „am Gleichgewichtspunkt des europäischen Spiels“ ${ }^{\text {‘7 }}$ zu platzieren und aus der Rolle als Juniorpartner Deutschlands zu befreien, die Hollande seinem Konkurrenten Sarkozy vorgeworfen hatte. Deutschland und Frankreich sollten, einem Präsidentenberater zufolge, wieder ein ausbalanciertes, nichtexklusives Paar bilden. ${ }^{48}$ Hollande selbst definierte die veränderte Rolle Frankreichs in Europa als „Bindeglied zwischen Nord- und Südeuropa“ - und damit ausdrücklich nicht als diejenige eines Anführers einer südeuropäischen Koalition gegen die Bundesrepublik und ihre nordeuropäischen Verbündeten. ${ }^{49}$ Es muss sich erst erweisen, ob es Frankreich mittels dieser flexibleren Koalitionsbildungen mit Partnerstaaten gelingen wird, seine Präferenzen zur Umgestaltung der Eurozone besser zur Geltung zu bringen. Der Preis dieser Strategie könnte in einem höheren Konfliktniveau der bilateralen Beziehungen zu Deutschland und einem Verlust an Entscheidungseffizienz auf europäischer Ebene liegen, wenn die wichtige bilaterale Verhandlungsebene weniger intensiv genutzt wird.

Die persönlichen Beziehungen zwischen Hollande und Merkel erreichen bislang nicht die Intensität der Kontakte zwischen Merkel und Sarkozy, die zu Beginn der Amtszeit Sarkozys allerdings ebenfalls deutlich distanzierter waren als zum Ende hin. ${ }^{50}$ Es ist durchaus vorstellbar, dass Situationen einer neuerlichen Krisenzuspitzung wieder zu einer Verdichtung bilateraler Abstimmungs- und Kompromissbildungsprozesse auf der ,Chefebene‘ führen.

Im Gegensatz zu ,Merkozy“ reisen Hollande und Merkel nicht mit vorab bilateral konzertierten Kompromissen in der Tasche zu Europäischen Ratstreffen. Im Oktober 2012 kam es zu einem abgestimmten deutsch-französischen Kompromiss zu strittigen Fragen der Bankenunion erst unmittelbar vor Beginn des Gipfels in Brüssel, im Vorfeld des Gipfels im Juni überhaupt nicht. Die Intensität bilateraler Konsultationen und Abstimmungsprozesse unterhalb der politischen Spitzenebene hat dem Vernehmen nach aber nicht nachgelassen. ${ }^{51}$ Davon zeugt auch die Einrichtung einer bilateralen Arbeitsgruppe zur Eurozonenkrise, die auf französischer Seite vom Direktor des Schatzamtes (Trésor), Ramon Fernandez, und auf deutscher Seite von Finanzstaatssekretär Thomas Steffen geleitet wird. ${ }^{52}$

Dass eine bilaterale Führungsrolle in der Europäischen Union im Rahmen der neuen politischen Konstellation denkbar und möglich ist, hat das proaktive und letztlich erfolgreiche Eintreten von Paris und Berlin für die Einführung einer Finanztransaktionssteuer gezeigt. Diese soll im Rahmen einer verstärkten Zusammenarbeit einer Teilgruppe von EU-Staaten verwirklicht werden. ${ }^{53}$

47 Vgl. Le Monde: Hollande-Merkel, l'histoire d'un couple sans exclusive, 23.-24. September 2012 (eigene Übersetzung).

48 Hollande sprach vor seiner Wahl auf einem SPD-Parteitag am 5.12.2012 von einer deutsch-französischen Freundschaft ,in Gleichheit und Respekt“. Vgl. SPD.de: Rede Francois Hollande auf dem SPD-Bundesparteitag am 5. Dezember 2011 in Berlin, 5.11.2012.

49 Süddeutsche Zeitung: Geliebte Baustelle, 2012.

50 Zum geschäftsmäßig-nüchternen Charakter der Beziehungen zwischen Hollande und Merkel. Vgl. Leo Klimm/ Claudia Kade: Merkel und Hollande: Partner - aber keine Freunde, in: FTD.de, 21.9.2012.

51 So die Aussage eines hohen Beamten der Europaabteilung des Bundesfinanzministeriums gegenüber dem Autor im Oktober 2012.

52 Vgl. Le Monde: Allemands et Français créent un groupe de travail sur la crise de la zone euro, 29.8.2012.

53 Vgl. Spiegel-Online: Elf EU-Länder wollen Finanzsteuer einführen, 9.10.2012. 
Mittelfristig wird Frankreich sein machtpolitisches Gewicht in der Europäischen Union und eine mit der Bundesrepublik gleichberechtigte Führungsrolle nur behaupten können, wenn es intern die dafür notwendigen und schmerzhaften Anpassungsleistungen erbringt. „Wenn sich das Ungleichgewicht [zwischen Frankreich und Deutschland] verschärft“, so Außenminister Laurent Fabius, ,,dann würde dies natürlich zu einem wirklichen Problem. “54

\section{Zeit der Entscheidungen}

Frankreich trieb die europäische Wirtschafts- und Währungsunion Anfang der 1990er Jahre nicht zuletzt aus machtpolitischen Motiven voran und sah darin ,,a means to redress partially the imbalance in the Franco-German relationship created by German economic power". ${ }^{55}$ Rund zwei Jahrzehnte später ist das Thema einer machtpolitischen Asymmetrie im Verhältnis zum deutschen Nachbarn aktueller denn je. Dies ist die Folge anhaltender Schwierigkeiten Frankreichs, sich den Herausforderungen des verschärften globalen und innereuropäischen Wettbewerbs und den Zwängen der Mitgliedschaft in einer Währungsunion durch eine kritische Prüfung und Reform seines Wirtschafts-, Sozial- und Wachstumsmodells zu stellen. Die Folgen einer erodierenden wirtschaftlichen Machtbasis für den europäischen Einfluss Frankreichs lassen sich durch eine geschickte europapolitische Strategiewahl nur partiell kompensieren. Der Schlüssel für eine solide Unterfütterung eines französischen Führungs- und Gestaltungsanspruchs in der Union und für konstruktive deutsch-französische Beziehungen der nächsten Jahre liegt in der erfolgreichen Rekonstruktion wirtschaftlicher Wettbewerbsfähigkeit und fiskalpolitischer Nachhaltigkeit. Beides ist auch von zentraler Bedeutung für die Stabilität der Eurozone. Die Herausforderung, vor die sich der neue Präsident und seine Regierungsmehrheit gestellt sehen, ist vergleichbar mit derjenigen, die Charles de Gaulle 1958 zu Beginn der V. Republik und François Mitterrand 1983 nach dem Scheitern des linkskeynesianischen Wirtschaftsexperiments zu bewältigen hatten. Beide haben letztlich erkannt, dass eine aktive außen- und europapolitische Rolle ihres Staates nur auf der Basis konsolidierter Staatsfinanzen und dank einer Wirtschaftspolitik möglich ist, die die relative Wettbewerbsfähigkeit und Wirtschaftskraft Frankreichs stärkt.

Hollande hat bisher sowohl in seiner Zeit als Parteivorsitzender der Sozialisten (1997-2008) wie auch in seinen ersten Monaten als Staatpräsident eher als Moderator, bisweilen als Zauderer und Zögerer gewirkt, und selten einen Führungsstil gezeigt, der dem von James MacGregor Burns beschriebenen Modell der „transforming leadership“ nahe käme. ${ }^{56}$ Angesichts der fragilen innenpolitischen Machtbasis und einer skeptischen öffentlichen Meinung muss Hollande jedoch eine ausgeprägte politische Führungsfähigkeit unter Beweis stellen und ein überzeugendes sozialliberales Reformnarrativ entwickeln, um die innenpolitische und wirtschaftliche Machtbasis einer aktiven und erfolgreichen Europapolitik zu rekonstruieren. Die wirtschaftliche Substanz hierzu besitzt das Land ohne Frage. Entscheidend ist seine politische Reformfähigkeit. Frankreich ist nicht der ,kranke Mann Europas'. Sollten Hollande und seine Linksregierung Ayrault die Reformherausforderungen jedoch nicht erfolgreich bewältigen, so könnte es aber dazu werden - mit unabsehbaren Risiken für die Eurozone und für die deutsch-französischen Beziehungen.

54 Vgl. Le Monde: Hollande-Merkel, 2012.

55 David J. Howarth: The French Road to European Monetary Union, Basingstoke 2001, S. 118.

56 James MacGregor Burns: Transforming Leadership: The New Pursuit of Happiness, New York 2003. 